\title{
Effects of Ankle Joint Exercise Programs with Hip Joint and Lumbopelvic Exercises on the Muscle Activity of Adults with Functional Ankle Instability
}

\author{
Jae-Ik Lee', Min-Bong Kang1, Jung-Hyun Ban', Young-Jae Jung ${ }^{1}$, Tae-Ho Kim²,* \\ ${ }^{1}$ Department of Rehabilitation Science, Graduate School, Daegu University, South Korea \\ ${ }^{2}$ Department of Rehabilitation Sciences, Department of Physiotherapy, Daegu University, South Korea
}

Received July 16, 2021; Revised August 20, 2021; Accepted September 21, 2021

\section{Cite This Paper in the following Citation Styles}

(a): [1] Jae-Ik Lee, Min-Bong Kang, Jung-Hyun Ban, Young-Jae Jung, Tae-Ho Kim, "Effects of Ankle Joint Exercise Programs with Hip Joint and Lumbopelvic Exercises on the Muscle Activity of Adults with Functional Ankle Instability," International Journal of Human Movement and Sports Sciences, Vol. 9, No. 6, pp. 1125 - 1130, 2021. DOI: 10.13189/saj.2021.090607.

(b): Jae-Ik Lee, Min-Bong Kang, Jung-Hyun Ban, Young-Jae Jung, Tae-Ho Kim (2021). Effects of Ankle Joint Exercise Programs with Hip Joint and Lumbopelvic Exercises on the Muscle Activity of Adults with Functional Ankle Instability. International Journal of Human Movement and Sports Sciences, 9(6), 1125 - 1130. DOI: 10.13189/saj.2021.090607.

Copyright $\bigcirc 2021$ by authors, all rights reserved. Authors agree that this article remains permanently open access under the terms of the Creative Commons Attribution License 4.0 International License

\begin{abstract}
In subjects with functional ankle instability, lumbopelvic instability causes instability of the hip and lower extremities. The purpose of this study is to determine whether an ankle exercise program with hip strengthening exercise and lumbopelvic stabilization exercise affects spine and ankle stability through muscle activity in adults with functional ankle instability. Thirty adults with functional ankle instability were randomly assigned to a hip joint strengthening exercise group and a lumbopelvic stabilization exercise group. The subjects participated in the designated program three times a week for 40 minutes a day. In the hip joint strengthening exercise group, a general hip joint strengthening exercise was used, and in the lumbopelvic stabilization exercise group, a lumbopelvic stabilization exercise using a sling was added. The intervention lasted 4 weeks, and both groups were instructed to refrain from other exercise during the 4-week intervention period. The muscle activity of the tibialis anterior, gluteus maximus, external oblique and contralateral latissimus dorsi was measured. Data were collected from the 30 patients. The results showed that the muscle activity of the tibialis anterior, gluteus maximus, lateral oblique muscle, and contralateral latissimus dorsi was significantly increased in both groups. This suggests that the existing hip joint strengthening exercise and lumbopelvic stabilization exercise are effective
\end{abstract}

approaches which not only help reduce hip joint pain and improve function, but also reduce functional ankle instability. Therefore, if an appropriate exercise program is provided for subjects with functional ankle instability, it is expected that it will help improve pain and function by reducing ankle instability and the frequency of re- injury.

Keywords Ankle Instability, Lumbopelvic Stabilization, Muscle Activity

\section{Introduction}

With the increasing population of people engaging in leisure activities and sports, damage related to sports has also been increasing. Ankle sprains account for $85 \%$ of the lateral ankle damages caused by ankle inversion. Among patients who have experienced sprained ankle joints, $10 \%$ to $50 \%$ experience chronic pain and re-injury symptoms, such as chronic ankle sprains [1].

In their daily lives, they cannot repair tissue weakness and pain-related damage, and thus inversion easily occurs [2].

Chronic ankle instability is classified into mechanical 
and functional instability. Mechanical ankle instability is caused by relaxation or impairment of ligaments, which are static stabilizing structures [3]. Freeman introduced the term "functional ankle instability" for the situation in which patients complain of joint instability due to incomplete recovery of damaged proprioception during acute ankle sprain [4]. Functional ankle instability reflects subjective instability, and related factor include impaired muscle strength and proprioception [5]. There can be weakness of the calf muscle on the outside of the ankle, decreased proprioception around the ankle joint [6], and decreased eccentric control of plantar flexion of the ankle joint [7]. The treatment of functional ankle instability includes proprioception exercises focusing on plantar flexion and dorsiflexion [8], ankle muscle strengthening methods focusing on the ankle joint, functional exercise methods related to agility [9], and kinetic taping [10]. It has also been reported that increasing the muscle activity of the peroneus longus and medial soleus muscles during treadmill walking using an auditory biofeedback input device is effective [11].

Ankle injury can also have a wider impact on other body functions. Changes in hip joint muscle activity and muscle strength have been reported in patients with chronic ankle instability [12]. Konradsen reported that the protective response of the muscles around the ankle appeared later than the moment of injury. To prevent repetitive damage due to ankle instability, it is important to control the hip muscle movement function and consider it when treating ankle joint instability [13]. The proximal control of the ankle complex affects the time in contact with the ground [14]. Van stated that when body alignment is changed due to an external factor applied to the foot, the interrelationship between the hip joint and spine ankle joint, and surrounding joints changes abnormally [15]. Zampagni reported that chronic ankle instability can cause changes in the muscles acting on the hip joint [16]. Furthermore, in a study of people with chronic ankle instability, Beckman and Buchanan used electromyography analysis to show that the activity of the hip muscles maintaining posture decreased [12]. These results are closely related to the causes of ankle sprains. Friel reported weakness of the hip joint muscle on the same side after chronic ankle sprain due to mediation [17].

Recently, research on the role of the hip joint muscles in the treatment and prevention of lower extremity injury has gained importance [18-20]. When ankle dysfunction is detected, it is vital to identify and treat the specific cause, and early treatment is necessary to prevent chronic ankle sprains [21]. These previous studies assert that continuous interaction between the ankle and hip muscles is necessary to maintain posture, balance, and center of gravity [22] and that hip joint muscle weakness can cause re-injury of the ankle.

Many studies have focused on the interrelationship between the hip and the lumbopelvic [23-26] which revealed the relationship between the hip joint and ankle instability. However, there are few studies on the relationship between the lumbar pelvis and ankle instability. Therefore, this study aimed to investigate whether an ankle exercise program with hip joint strengthening exercises and lumbopelvic stabilization exercises as an exercise method for resolving symptoms caused by functional ankle instability affects the muscle activity of subjects with functional ankle instability.

\section{Methods}

\subsection{Subjects}

The subjects of this study were 30 adults with functional ankle instability. They included students enrolled in D University, in Daegu, and people belonging to an athletic club. Subjects were those who had an ankle instability instrument score of 4 or higher, a percentile of $90 \%$ or less on the foot and ankle ability measure, persistent anxiety and functional decline in the ankle joint, and recurrent ankle joint damage. The excluded were those who had a history of surgery on the lower extremities, balance disorders, exercise or treatment experiences that had affected their balance within the last month, and paresthesia or muscle paralysis due to neurological disorders. All subjects were fully informed about purpose and method of the study before the experiment was conducted, and the experiment was performed after they agreed to voluntarily participate. The general characteristics of the subjects are shown in Table 1.

Table 1. The general characteristics of the subjects

\begin{tabular}{cccc}
\hline & STR & STA & $\boldsymbol{p}$ \\
\hline Age(years) & $30.93 \pm 5.44^{\mathrm{a}}$ & $31.13 \pm 2.89$ & 0.100 \\
Height $(\mathrm{cm})$ & $171.86 \pm 5.74$ & $170.60 \pm 7.02$ & 0.755 \\
Weight $(\mathrm{kg})$ & $73.26 \pm 12.20$ & $70.73 \pm 14.15$ & 0.748 \\
BMI & $24.71 \pm 3.32$ & $24.13 \pm 3.68$ & 0.612 \\
\hline
\end{tabular}

a Mean \pm Standard deviation

STR: Exercise program group performing hip joint strengthening exercises.

STA: Exercise program group performing lumbopelvic stabilization exercises.

\subsection{Assessment}

Eight-channel wireless surface electromyography (TeleMyo DTS, Noraxon Ins, AZ, USA) was used to measure the activity of the tibialis anterior, gluteus maximus, external abdominal oblique, and contralateral latissimus dorsi muscles (Figure 1). The signals of each 
muscle were measured with maximum voluntary isometric contractions. Measurement posture of the maximum voluntary isometric contraction was carried out based on the method presented by Cram [26].

The maximum voluntary isometric contraction of each muscle was measured three times for $5 \mathrm{~s}$ each, and then the average signal for the intermediate $3 \mathrm{~s}$ was used (not the initial and last 1s). To minimize the muscle fatigue that may have occurred due to repeated measurements, there was 1 min of rest between exercises.

The anterior tibialis anterior electrode was attached to the outer $2 \mathrm{~cm}$ area on the line of the anterior tibia. The gluteus maximus electrode was attached parallel to the muscle at the midpoint between the great femur and the second sacral vertebra. The external oblique electrode was attached to the line connecting the lower rib below the thoracic cage and the contralateral rib cage. Electrodes of the latissimus dorsi were attached $4 \mathrm{~cm}$ below the inferior angle of the scapula [27].

\subsection{Experimental Procedure}

The 30 participants were randomly assigned to either the ankle exercise program group with hip joint strengthening exercise $(n=15$, hip joint strengthening exercise group) or the ankle exercise program group with lumbopelvic stabilization exercise $(\mathrm{n}=15$, lumbopelvic stabilization exercise group). Muscle activity of all subjects was measured before and after the intervention. The exercises were conducted by a physical therapist with more than five years of clinical experience. The exercise duration was the same in each group: 40 min a day, 3 days a week, for 4 weeks. Each session consisted of $5 \mathrm{~min}$ of warm-up, $30 \mathrm{~min}$ of main exercise $(5 \mathrm{~min}$ for each exercise), and $5 \mathrm{~min}$ of finishing exercise. The exercise programs of two groups were designed with reference to the methods of previous studies [28-30]. For the warm-up, the subjects walked on a treadmill for $5 \mathrm{~min}$. The main exercise consisted of six exercises. Dorsiflexion, ankle sprain, split lunge, and squat (Figures 1 and 2) were used in both groups, and the remaining two exercises were different. The hip joint strengthening exercise group performed a hip extension exercise in the prone position and a hip abduction exercise in the side-lying position (Figure. 3). Three 3 sets, with- 15 repetitions per set, were performed. Each week, considering the condition of the subject, the difficulty was increased using an elastic band (Hygenic $®$, USA). The lumbopelvic stabilization exercise group performed a supine pelvic lift and side-lying hip abduction with a sling as presented in Gitle Kirkesola's neurac method (Figure 4) [31]. Three sets, with 15 repetitions per set, were performed. The finishing exercise comprised simple stretching and deep breathing. After each set, the rest period was 30s.

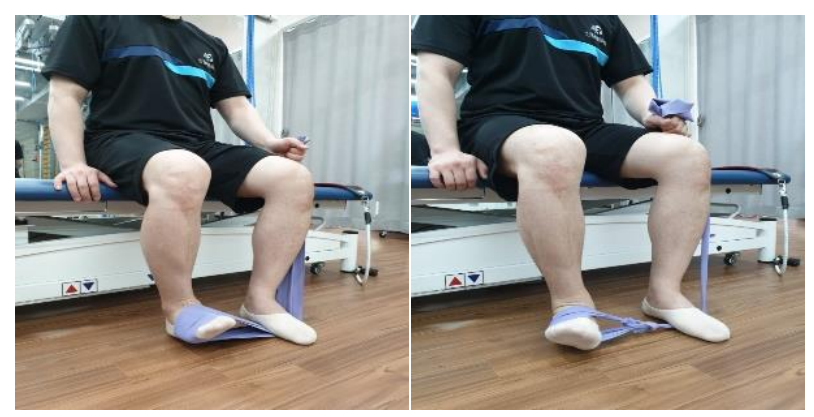

Figure 1. Ankle strengthening exercises

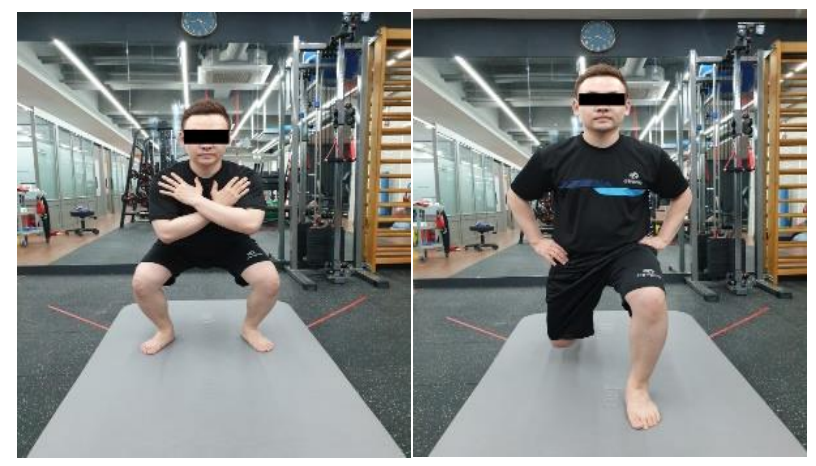

Figure 2. Lower limb strength exercises

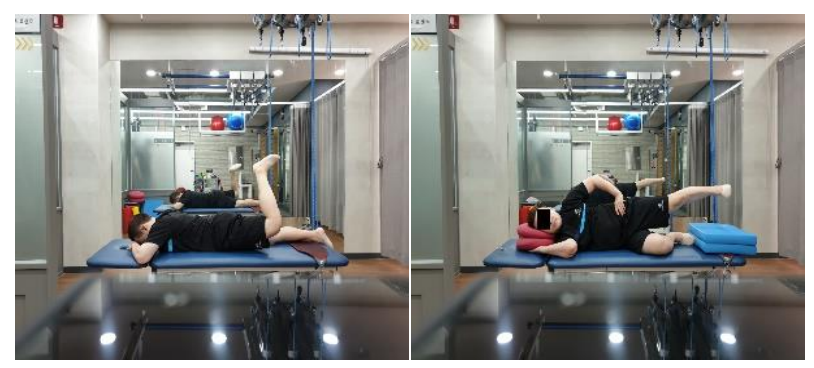

Figure 3. Hip strengthening exercise

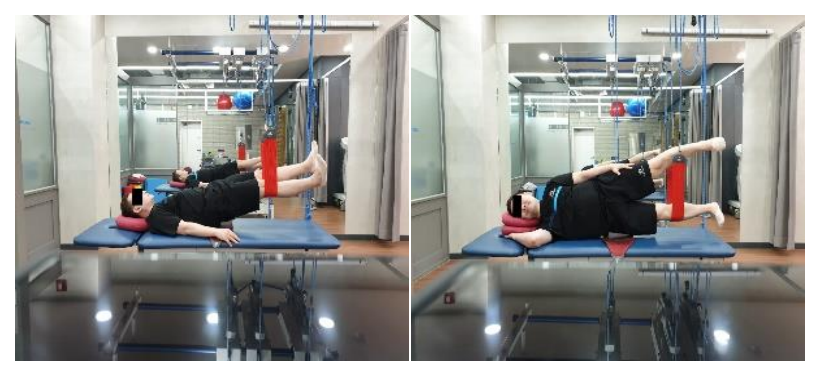

Figure 4. Lumbopelvic stabilization exercise

\section{Statistical Analysis}

The data measured in this study were analyzed using the Statistical Package for the Social Sciences (SPSS) version 20.0 for Windows (SPSS Inc., Chicago). The Kolmogorov-Smirnov test was used for a test of normality. The results of the two groups were compared, and the statistical significance of the difference was determined by performing an independent $t$-test. A paired 
t-test was performed to compare the within-group differences between the before- and after-exercise results. The significance level was set as 0.05 .

\section{Results}

After four weeks of intervention, the tibialis anterior, gluteus maximus, external abdominal oblique, and contralateral latissimus dorsi muscle activity of both groups significantly increased $(\mathrm{p}<0.05)$ (Table 2). When the two groups were compared, there was no significant difference in the muscle activity values between the hip joint strengthening exercise group and the lumbopelvic stabilization exercise group after four weeks of exercise $(\mathrm{p}>0.05)$ (Table 2).

\section{Discussion}

The purpose of this study was to compare changes in muscle activity by applying an ankle exercise program with hip joint strengthening exercises and lumbopelvic stabilization exercises to subjects with functional ankle instability for four weeks. Ankle exercises are used to strengthen foot stability. In addition to these methods, hip joint and lumbopelvic stabilization exercises were individually performed and studied to compare the effects of each method on ankle stabilization. It was found that the hip joint strengthening exercise group had significantly higher post-intervention EMG values compared to the pre-intervention values. However, there was no significant difference between the groups.

In cases of ankle instability, the ankle eversion muscle is weakened [9], and there is hip and lumbopelvic muscle weakening [12,32]. The tibialis anterior functions to prevent the foot from being dragged through the dorsiflexion while walking and to prevent the inversion, or plantar flexion, which is a posture that can lead to ankle sprain. In a study on the effect of neuromuscular control training for ankle instability on dynamic balance ability, the muscle activity of the anterior tibialis anterior significantly increased after the intervention [33], just as in our study. The gluteus maximus is a typical hip extensor. This muscle stabilizes the lower back by sharing the thoracolumbar fascia with the contralateral broad latissimus dorsi. The external abdominal oblique, together with the contralateral hip adductor muscle, works to stabilize the lumbopelvic region. Our results agree with the study that showed increased muscle activity of the gluteus maximus after squats and lunges were performed by subjects with chronic ankle instability [34].

Table 2. The comparison of muscle activity during maximal voluntary contraction

\begin{tabular}{|c|c|c|c|c|c|c|}
\hline & Group & Before & after & Difference value & $\mathbf{t}$ & $p$ \\
\hline \multirow{4}{*}{$\begin{array}{c}\text { Tibialis } \\
\text { anterior } \\
(\mu \mathrm{V})\end{array}$} & STR & $331.08 \pm 141.84^{\mathrm{a}}$ & $427.26 \pm 142.47$ & $96.24 \pm 46.50$ & -4.350 & $0.001^{*}$ \\
\hline & STA & $307.74 \pm 54.00$ & $419.79 \pm 69.90$ & $112.05 \pm 49.59$ & -6.105 & $0.000^{*}$ \\
\hline & $\mathrm{t}$ & & & -0.406 & & \\
\hline & $p$ & & & 0.688 & & \\
\hline \multirow{4}{*}{$\begin{array}{l}\text { Gluteus } \\
\text { maximus } \\
(\mu \mathrm{V})\end{array}$} & STR & $229.68 \pm 64.68$ & $319.08 \pm 80.97$ & $89.34 \pm 42.90$ & -4.088 & $0.001^{*}$ \\
\hline & STA & $255.30 \pm 61.80$ & $357.18 \pm 70.05$ & $101.88 \pm 50.61$ & -15.596 & $0.000^{*}$ \\
\hline & $\mathrm{t}$ & & & 0.156 & & \\
\hline & $p$ & & & 0.877 & & \\
\hline \multirow{4}{*}{$\begin{array}{c}\text { External } \\
\text { abdominal } \\
\text { oblique } \\
(\mu \mathrm{V})\end{array}$} & STR & $162.03 \pm 64.11$ & $230.76 \pm 67.26$ & $68.70 \pm 29.07$ & -4.772 & $0.000^{*}$ \\
\hline & STA & $205.47 \pm 56.22$ & $297.99 \pm 68.54$ & $74.55 \pm 36.93$ & -5.807 & $0.000^{*}$ \\
\hline & $\mathrm{t}$ & & & 0.571 & & \\
\hline & $p$ & & & 0.573 & & \\
\hline \multirow{4}{*}{$\begin{array}{l}\text { Opposite } \\
\text { latissimus } \\
\text { dorsi } \\
(\mu \mathrm{V})\end{array}$} & STR & $241.05 \pm 50.52$ & $309.57 \pm 69.12$ & $68.49 \pm 51.03$ & -3.878 & $0.002^{*}$ \\
\hline & STA & $255.48 \pm 48.90$ & $338.97 \pm 59.34$ & $83.49 \pm 39.06$ & -6.727 & $0.000^{*}$ \\
\hline & $\mathrm{t}$ & & & -1.617 & & \\
\hline & $p$ & & & 0.117 & & \\
\hline
\end{tabular}

${ }^{\mathrm{a}}$ Mean \pm Standard deviation

STR: Exercise program group performing hip joint strengthening exercises

STA: Exercise program group performing lumbopelvic stabilization exercises 
The muscle activity value was significantly increased in both groups before and after the exercise intervention in the intragroup comparison, but there was no significant difference in the group comparison. No statistically significant difference was found. Therefore, it is thought that both the hip joint strengthening exercises and the lumbopelvic stabilization exercises increased the muscle activity of the lateral oblique, gluteus maximus, contralateral latissimus dorsi and tibialis anterior. The stability of the ankle and trunk was increased because every intervention increased the activity of the muscles that contributed to the stabilization of the ankle and trunk.

The limitation of this study is that the data could not be normalized because only the MVIC value was measured and not the EMG value during walking. In consideration of this limitation, future research on exercise programs for subjects with functional ankle instability should provide more objective evidence through data that are normalized by the measurement of EMG values of each muscle while walking. In addition to the use of electromyography, it is necessary to develop an exercise program that reduces ankle instability symptoms through an approach that include ankle function and balance and it is also necessary to improve one-legged function, which is an essential element for walking and running.

\section{Acknowledgement}

This research was supported by Daegu University Future Scholars Program, 2021

\section{REFERENCES}

[1] P. Sperryn, "Sports medicine on the line?," Br J Sports Med, 28(1):3, 1994.

[2] J. Hertel, "Functional instability following lateral ankle sprain," Sports Med, 29(5):361-371, 2000.

[3] D. W. Kim, K. S. Sung, "Chronic Lateral Ankle Instability," Journal of Korean Foot \& Ankle Society, 22(2):55-61, 2018.

[4] M. A. Freeman, M. R. Dean, I. W. Hanham, "The etiology and prevention of functional instability of the foot," $J$ Bone Joint Surg Br, 47(4):678-685, 1965.

[5] DiGiovanni CW, Brodsky A. Current concepts: lateral ankle instability. Foot Ankle Int. 2006;27(10):854-866.

[6] T. Willems, Witvrouw, J. Verstuyft, P. Vaes, D. De Clercq, "Proprioception and Muscle Strength in Subjects with a History of Ankle Sprains and Chronic Instability," $J$ Athl Train, 37(4):487-493, 2002.

[7] J. Fox, C. L. Docherty, J. Schrader, T. Applegate, "Eccentric plantar-flexor torque deficits in participants with functional ankle instability," J Athl Train, 43(1):51-54, 2008.
[8] C. M. Bleakley, S. R. O'Connor, M. A. Tully, "Effect of accelerated rehabilitation on function after ankle sprain: randomised controlled trial," BMJ, 10;340:c1964, 2010.

[9] C. W. Lin, C. E. Hiller, R. A. de Bie, "Evidence-based treatment for ankle injuries: a clinical perspective," J Man Manip Ther, 18(1):22-28, 2010.

[10] C. de-la-Torre-Domingo, I. M. Alguacil-Diego, F.Molina-Reuda, "Effect of Kinesiology Tape on Measurements of Balance in Subjects With Chronic Ankle Instability: A Randomized Controlled Trial," Arch Phys Med Rehabil, 96(12):2169-2175, 2015.

[11] L. Donovan, M. A. Feger, J. M. Hart, "Effects of an auditory biofeedback device on plantar pressure in patients with chronic ankle instability," Gait Posture, 44:29-36, 2016.

[12] S. M Beckman, T. S. Buchanan, "Ankle inversion injury and hypermobility: effect on hip and ankle muscle electromyography onset latency," Arch Phys Med Rehabil, 76(12):1138-1143, 2015.

[13] L. Konradsen, M Voigt, C. Højsgaard, "Ankle inversion injuries The role of the dynamic defense mechanism," Am J Sports Med, 25(1):54-58, 1997.

[14] S. Robbins, E. Waked, "Factors associated with ankle injuries Preventive measures," Sports Med, 25(1):63-72, 1998.

[15] S. Van Deun, F. F. Staes, K. H. Stappaerts,"Relationship of chronic ankle instability to muscle activation patterns during the transition from double-leg to single-leg stance," Am J Sports Med, 35(2):274-281, 2007.

[16] M. L. Zampagni, I. Corazza, A. P. Molgora, M. Marcacci, "Can ankle imbalance be a risk factor for tensor fascia lata muscle weakness?," J Electromyogr Kinesiol, 19(4):651-659, 2009.

[17] K. Friel, N. McLean, C. Myers, M. Caceres, "Ipsilateral hip abductor weakness after inversion ankle sprain," J Athl Train, 41(1):74-78, 2006.

[18] M.K. Dwyer, S. N. Boudreau, C. G. Mattacola, "Comparison of lower extremity kinematics and hip muscle activation during rehabilitation tasks between sexes," J Athl Train, 45(2):181-190, 2010.

[19] W. T. Lim, "Tensile force transmission from the upper trunk to the contralateral lower leg throughout the posterior oblique sling system," International journal of Human Movement and Sports Sciences, 9(2): 294-300, 2021. DOI: 10.13189/saj.2021.090217.

[20] A. H. Mahmoud, "The effect of a training program using speed-specific strength exercises on sensory-motor perception in learning stroke forehand smash in badminton material for students of the Hashemite university," International journal of Human Movement and Sports Sciences, 8(6): 299-307, 2020. DOI: 10.13189/saj.2020.080601.

[21] J. N. Bernier, D. H. Perrin DH, "Effect of coordination training on proprioception of the functionally unstable ankle," J Orthop Sports Phys Ther, 27(4):264-275, 1998.

[22] M. Y. Lim, M. S. Park, S. G. Lim, "Hip and ankle strength in taekwondo athletes with chronic ankle instability," 
Exercise Science. 19(4):371-380, 2010.

[23] M. K. Chan, K. W. Chow, A. Y. Lai, N. K. Mak, J. C. Sze, S. M. Tsang, "The effects of therapeutic hip exercise with abdominal core activation on recruitment of the hip muscles," BMC Musculoskelet Disord, 18(1):313, 2017.

[24] S. L. Hoffman, M. B. Johnson, D. Zou, "Effect of classification-specific treatment on lumbopelvic motion during hip rotation in people with low back pain," Man Ther, 16(4):344-350, 2011.

[25] J. A. Smith, J. M. Jr. Popovich, K. Kulig, "The influence of hip strength on lower-limb, pelvis, and trunk kinematics and coordination patterns during walking and hopping in healthy women," J Orthop Sports Phys Ther, 44(7):525-531, 2014.

[26] T. H. Kim, W. M. Je. B. H. Hwang, "Effects of pelvic compression belt on three dimensional motions of pelvic and trunk during one leg standing," J Musculoskelet Sci Technol, 3(1):22-25, 2019.

[27] J. R. Cram, G. S. Kasman, J. Holtz, "Electrode placement. Introduction to Surface Electromyography," Gaithersburg, MD: Aspen Publishers 1998.

[28] S. A. Hale, J. Hertel, L. C. Olmsted-Kramer, "The effect of a 4-week comprehensive rehabilitation program on postural control and lower extremity function in individuals with chronic ankle instability," J Orthop Sports Phys Ther, 37(6):303-311, 2007.
[29] D. M. Selkowitz, G. J. Beneck, C. M Powers, "Which exercises target the gluteal muscles while minimizing activation of the tensor fascia lata?," Electromyographic assessment using fine-wire electrodes, J Orthop Sports Phys Ther. 43(2):54-64, 2013.

[30] T. Wagner, N. Behnia, W. K. Ancheta, R. Shen, S. Farrokhi, C. M. Powers, "Strengthening and neuromuscular reeducation of the gluteus maximus in a triathlete with exercise-associated cramping of the hamstrings," J Orthop Sports Phys Ther, 40(2):112-119, 2010.

[31] G. Kirkesola, "Neurac-a new treatment method for long-term musculoskeletal pain," $J$ Fysioterapeuten, 76:16-25, 2009.

[32] Y. W. Kim, T. H. Kim, M. N. Yang, Y. S. Yon, J. H. Lee, "comparison of activities of tibialis anterior peroneus longus, and tibialis posterior muscles according to lunge squats and bulgarian split squats in a healthy population," $\mathrm{J}$ KEMA, 1(1):26-30, 2017.

[33] F. Pozzi, M. Moffat, G. Gutierrez "Neuromuscular control during performance of a dynamic balance task in subjects with and without ankle instability," Int J Sports Phys Ther, 10(4):520-529, 2015.

[34] K. A. Webster, P. A. Gribble, "A comparison of electromyography of gluteus medius and maximus in subjects with and without chronic ankle instability during two functional exercises," Phys Ther Sport, 14(1):17-22, 2013. 